\title{
Cal, un antiguo material como una renovada opción para la construcción
}

\author{
Lime, an Ancient Material as a Renewed Option \\ for Construction
}

\author{
Galván-Ruiz M. \\ División de Investigación y Posgrado \\ Facultad de Ingeniería \\ Universidad Autónoma de Querétaro, Qro. \\ E-mail:mgr@uaq.mx \\ Velázquez-Castillo R. \\ División de Investigación y Posgrado \\ Facultad de Ingeniería \\ Universidad Autónoma de Querétaro, Qro. \\ E-mail: rodrigo.velazquez@uaq.mx
}

Información del artículo: recibido: mayo de 2007, reevaluado: marzo de 2010, aceptado: agosto de 2010

\section{Resumen}

En esta investigación se estudiaron las transiciones de los materiales del ciclo de la cal. Se obtuvieron muestras de carbonato de calcio de un banco en los alrededores de Bernal, Querétaro. El carbonato de calcio es la materia prima para obtener óxido de calcio después de un proceso de calcinación e hidróxido de calcio, luego de una hidratación. Como etapa final del ciclo de la cal, el hidróxido de calcio interactúa con el bióxido de carbono del aire para formar nuevamente carbonato de calcio. Los cambios durante el ciclo se describen a través de difracción de rayos $\mathrm{X}$ por polvos para la identificación de las fases cristalinas. Microscopía electrónica de barrido fue aplicada para revisar la morfología, la topología, la estructura y el hábito de crecimiento cristalino. Para obtener la distribución de tamaños de partículas del hidróxido de calcio, se empleó dispersión dinámica de luz. Se empleó espectroscopía infrarroja por transformada de Fourier para identificar moléculas y enlaces atómicos. Estos resultados son una contribución al campo de la industria de construcción, tanto como un punto de partida a nuevas aplicaciones del hidróxido de calcio para la ingeniería actual, como en procesos de conservación y restauración del patrimonio histórico.

\section{Descriptores}

- carbonato de calcio

- óxido de calcio

- hidróxido de calcio

- caracterización físico-química

- construcción

- patrimonio histórico 


\begin{abstract}
In this research the transitions of the materials of the cycle of lime were studied. Samples of calcium carbonate were obtained from a bank at the surroundings of Bernal, Querétaro. The calcium carbonate is the raw material to obtain calcium oxide after a process of calcination, and calcium hydroxide following a hydration. As a final stage of the lime cycle, the calcium hydroxide interacts with the carbon dioxide of the air to form calcium carbonate all over again. The changes during the cycle were described through X-ray diffraction by powders, for the identification of the crystalline phases. Scanning electron microscopy was done to review the morphology, topology, structure and crystalline growth habit. To obtain the particles sizes distribution of the calcium hydroxide, dynamic dispersion of light was used. Fourier transformed infrared spectroscopy was done to identify molecules and atomic connections. These results are a contribution to the field of the construction industry, as much as a starting point to new applications of the calcium hydroxide for the current engineering activities, and in conservation and restoration processes of the historical patrimony.
\end{abstract}

\section{Introducción}

El mundo fue construido con cal, la importancia histórica de la cal en la construcción se encuentra ampliamente documentada (Cazalla et al., 2000. Arandigoyen et al., 2006. Moropoulou et al., 2001, 2003 y 2004). Los morteros de cal fueron utilizados en muchas de las grandes estructuras por civilizaciones más tempranas, pero los romanos fueron los primeros en usarlos ampliamente en edificación, desarrollando métodos de fabricación, en general, con base en cementante natural de cal y estableciendo técnicas de construcción apropiadas (Delatte, 2001). En el siglo xviII, los trabajos de Smeaton condujeron al conocimiento científico sobre las propiedades de la cal hidráulica, lo que derivó en la tecnología y la patente del cemento Pórtland (Cowper 1927, reprinted 2000). Desde el siglo xix hasta hoy, la aparición del cemento Portland ordinario (OPC, por sus siglas en inglés) produjo un decrecimiento importante en el uso de la cal en la construcción, porque el OPC brinda un endurecimiento inicial más rápido y mayor resistencia a edades tempranas (Hill et al., 1992). Aunque éstas no son las únicas características deseables en un cementante, muchos beneficios pueden derivarse del retorno al uso apropiado de cal en la industria de la construcción, entre otros: su producción requiere temperaturas inferiores a la del OPC y menos energía durante su calcinación implica menor emisión de gases de efecto invernadero y mínima contracción, lo que evita agrietamientos; los morteros de cal resisten algún grado de acomodamiento en la mampostería, mientras los basados en OPC carecen de tolerancia a los movimientos; el deterioro causado por los álcalis contenidos en el OPC y que no presentan este problema en los mor- teros de cal; menor conductividad térmica que hace a las habitaciones más confortables; mayor impermeabilidad y mejor adherencia para materiales de mampostería comparado con productos basados en OPC (Shi, 2001). Los morteros de cal son materiales dinámicos, continúan interactuando con el ambiente después de endurecer durante el proceso de carbonatación y se adaptan a los cambios en las estructuras (Holmes et al., 2003). El endurecimiento por carbonatación se produce cuando el contenido de humedad ha disminuido lo suficiente y el $\mathrm{Ca}(\mathrm{OH})_{2}$ reacciona con el $\mathrm{CO}_{2}$ del aire formando $\mathrm{CaCO}_{3}$. Este proceso no puede realizarse bajo el agua; la carbonatación ocurre lentamente desde el exterior hacia el interior del mortero. Las diferencias crecientes de los precios del combustóleo y otros combustibles usados por los fabricantes de cal son afectados directamente por el mercado internacional del petróleo, que contribuye a una mayor incertidumbre y volatilidad en los precios; de acuerdo con datos de la Asociación Nacional de Fabricantes de Cal (ANFACAL) la producción nacional anual de cal es de 6 000,000 de toneladas, mientras que el mercado demanda $4^{\prime} 000,000$ de toneladas; esta industria mexicana vive una crisis propiciada por un mercado ofertado y costos crecientes, por lo que requiere cambios para su supervivencia. El incremento de calidad y la diversificación de productos con valor agregado a partir de cal como materia prima, significan opciones para la viabilidad de empresas y empleos en México. Esta investigación es un punto de partida para el mejoramiento de la cal para su uso en la construcción y en otras aplicaciones; por lo que se caracterizaron las muestras mediante difracción de rayos X (XRD); microscopía electrónica de barrido (SEM); espectroscopía infrarroja por transformada de Fourier 
(FTIR); espectrometría de masas por inducción de plasma acoplado (ICP-MS) y dispersión dinámica de luz (DLS). En el ciclo de la cal, la piedra caliza $\left(\mathrm{CaCO}_{3}\right)$ constituye en su estado natural el punto de partida obligado para la producción de la cal. Una vez extraída, se lleva a una instalación de trituración primaria donde se reduce hasta un tamaño máximo de entre 80 y 200 $\mathrm{mm}$. La obtención de una u otras granulometrías varía en función del tipo y diseño del horno empleado (verticales, horizontales, y rotatorios). En la siguiente etapa, la calcita es calcinada a una temperatura de entre $900 \mathrm{y}$ $1200^{\circ} \mathrm{C}$ y luego, mediante transportes adecuados, la cal viva obtenida se lleva a silos de almacenamiento, desde donde puede ser distribuida en sacos o a granel. La siguiente etapa del proceso es la producción de cal hidratada o apagada; la cal viva se lleva al hidratador, donde se mezcla con la cantidad adecuada de agua para satisfacer su afinidad higroscópica. El ciclo se cierra con la carbonatación, reacción del hidróxido de calcio con el dióxido de carbono del aire, con lo que regresa a un estado análogo al punto de partida como $\mathrm{CaCO}_{3}$. En la actualidad existen varias marcas de cal comercial para la construcción que provienen de diferentes canteras, lo que las hace heterogéneas. La cal de los productores mexicanos afiliados a la ANFACAL está regulada por la Norma Mexicana NMX-C-003-ONNCCE-1996, que establece que la cal hidratada para la construcción se clasifica en cuanto a su composición química en tipo único y en un mismo grado de calidad. Las marcas comerciales más populares en México son regularmente las disponibles en cada región, pero sobresalen: El Tigre, Calfina, Perla, Incalpa, Cobra, Muro, Huescalapa, Santa Fe, Polar, Noroeste, Santa Emilia, Banderilla, Nachón, Teziutecas, Santa Cruz, Calizas Universales, Tehuacán, Maria Luisa, Santa María, Gloria, Guadalajara, Hidracán, San Lorenzo, Tirado, Nay, Colimán, Pet-Cal, Petrocal, El Piñón, Torreón, Chiapas, Panini, El Refugio, Oax, Santa Lucía, Aguascalientes, San Martín, Azteca, La Tehuana, Calfin, Calfort, Los Arcos, Maciel, Teloloapan, Zapotiltic, Calteco, Diamante, San Antonio, Carlan, Duracal, Titán, Mitza, BYM, Balún Canán, Del Valle,
Calidra, Porter, Marfil, Suprema, Tolucal, Valasa, Tobora, Edifical, Tepeyahualco, Topo, Calhifornia, Icor, y otras; diseminadas en el territorio mexicano incluidos algunos estados no productores (Chihuahua, Durango, Tlaxcala, y Nayarit), han sido indistintamente empleadas a lo largo de muchas décadas con resultados satisfactorios. En algunos casos podrían presentarse impurezas; en la tabla 1 se describen las muestras: SA1 calcita proveniente de la cantera, SA2 óxido de calcio y finalmente SA3 muestra de hidróxido de calcio.

\section{Materiales y métodos}

\section{Muestreo}

Se estudiaron tres muestras identificadas como sigue: SA1corresponde a $\mathrm{CaCO} 3, \mathrm{SA} 2 \mathrm{a} \mathrm{CaO}, \mathrm{y} \mathrm{SA} 3$ a $\mathrm{Ca}(\mathrm{OH})_{2}$. La muestra SA1 fue obtenida en una cantera de piedra caliza luego de trazar una retícula, recolectando tres muestras de aproximadamente $250 \mathrm{~g}$ de doce posiciones aleatorias en casillas de la retícula, mismas que se mezclaron y dividieron por cuartos hasta obtener una muestra representativa de la totalidad. La muestra SA2 (cal viva) fue obtenida en la boca del horno de calcinación, siguiendo un procedimiento de muestreo equivalente al empleado para la obtención de SA1. Finalmente, la muestra SA3 se obtuvo en la boca de salida del equipo de hidratación de una empresa fabricante de cal, con muestreo semejante al citado. Las muestras se aislaron del ambiente para su conservación.

\section{Difracción de rayos-X (XRD)}

Con el propósito de identificar las fases cristalinas presentes, las muestras se analizaron por medio de la técnica de difracción de rayos $X$ por polvos, empleando un difractómetro Bruker AXS modelo D8 Advance (Alemania), que utiliza la emisión $\mathrm{K} \alpha$ del cobre de 0.154 $\mathrm{nm}$. Las condiciones de operación del equipo fueron a $35 \mathrm{kV}$ de voltaje de aceleración y $30 \mathrm{~mA}$, con una velocidad de barrido de $2^{\circ} / \min (2 \theta)$ y un tiempo de paso de

Tabla 1. Muestras de carbonato, óxido e hidróxido de calcio

\begin{tabular}{ccc}
\hline Muestra & Origen & Descripción \\
\hline SA1 & $\begin{array}{c}\text { Banco único de piedra caliza de Cal San Antonio en } \\
\text { Tolimán, Qro. (en la vecindad de Bernal) }\end{array}$ & $\begin{array}{c}\text { Piedra caliza cruda triturada y cribada, representativa de la } \\
\text { producción de la fecha de muestreo en el banco único. }\end{array}$ \\
SA2 & $\begin{array}{c}\text { Banda transportadora de salida del horno de Cal San } \\
\text { Antonio en Tolimán, Qro. }\end{array}$ & $\begin{array}{c}\text { Cal viva recién calcinada con combustóleo, representativa } \\
\text { de la producción en la fecha de muestreo. }\end{array}$ \\
SA3 & $\begin{array}{c}\text { Almacén de producto comercial a granel previo a su } \\
\text { ensacado, Cal San Antonio en Tolimán, Qro. }\end{array}$ & $\begin{array}{c}\text { Cal hidratada en polvo, representativa de la producción en } \\
\text { la fecha de muestreo. }\end{array}$ \\
\hline
\end{tabular}


$0.6 \mathrm{~s}$, en un intervalo de medición de $2^{\circ}$ a $70^{\circ}(2 \theta)$, a temperatura ambiente de $21^{\circ} \mathrm{C}$.

\section{Microscopía electrónica de barrido (SEM)}

La microestructura, morfología y topología de las muestras SA1, SA2 y SA3 se observaron empleando un microscopio electrónico de barrido (Jeol JSM 5600, Japón), utilizando electrones secundarios para formar las imágenes. Las condiciones de operación en el microscopio para la observación de las muestras fueron de $20 \mathrm{kV}$ de voltaje de aceleración, alto vacío ( $10^{-3}$ Torr) y a las muestras se les aplicó un recubrimiento de película delgada de oro por medio de un sputtering.

\section{Espectroscopía infrarroja por transformada} de Fourier (FTIR)

La identificación química de las sustancias constitutivas de las muestras se realizo a través de FTIR. Las muestras se molieron hasta obtener polvos finos, los cuales se combinaron con polvo de bromuro de potasio hasta tener una mezcla homogénea. Los análisis se llevaron a cabo utilizando un espectrofotómetro Bruker modelo Vector 33 FT-IR (EEUU), por medio de la técnica de reflectancia difusa. Además del análisis de los materiales, se estudiaron muestras de estándares como referencia: $\mathrm{CaCO}_{3}$ (Baker analyzed 99.9\%), $\mathrm{CaO}$ (Fermont, $96.9 \%$ ) y $\mathrm{Ca}(\mathrm{OH})_{2}$ (Fermont, 96.4\%) empleando las mismas condiciones citadas.

Espectrometría de masas por inducción de plasma acoplado (ICP-MS)

Un análisis elemental de los materiales se llevó a cabo por medio de ICP-MS. Esta técnica permite un análisis de alta cuantitatividad, el cual permite la cuantificación de las impurezas presentes. Para ello se empleó un espectrómetro de masas (Thermo Elemental Mass system $\mathrm{X}$-series ICP-MS, Standard Sample Introduction System, USA).

\section{Dispersión dinámica de luz (DLS)}

La distribución de tamaños de partículas del hidróxido de calcio (muestra SA3) se determinó mediante un equipo de dispersión dinámica de luz Brookhaven Instruments (USA) modelo BI-APD que utiliza un láser rojo con longitud de onda de $632.8 \mathrm{~nm}$ a un ángulo de $90^{\circ}$, tiempo de retraso $5 \mu \mathrm{s}$, temperatura ambiente $23^{\circ} \mathrm{C}$ y dilución en agua bi-destilada; programa de análisis cumulantes non negative list square. La primera medi- ción se hizo inmediatamente luego de una agitación vigorosa; la segunda a los 15 min y una tercera después de media hora.

\section{Resultados y discusión}

La Norma Mexicana NMX-C-003-ONNCCE-1996 establece con relación a la cal hidratada: tipo único, $80.00 \%$ mínimo de contenido de óxido de calcio, y máximo $5.00 \%$ de óxido de magnesio. Se trata de una norma de carácter voluntario, por lo que en el mercado mexicano es poco común su cumplimiento. Muchas de las empresas mexicanas fabricantes de cal hidratada para construcción, procuran reducir sus costos a través de argucias, como agregar los residuos de corazones de carbonato de calcio que no alcanzaron la transformación en óxido de calcio durante su tránsito por el horno, finamente molidos, así como otros agregados de coloración blanquecina susceptibles de molienda. Comparativamente, la molienda de inertes es mucho más barata comparada con la calcinación, pero demerita la pureza de la cal hidratada comercial e incuestionablemente, sus propiedades como cementante. La variación de contenidos de hidróxido de calcio en cada marca es abrumado$\mathrm{ra}$, es decir, la norma de referencia generalmente no se respeta, lo que se traduce en un mercado totalmente incierto en cuanto a la calidad de lo que el usuario final supone que adquiere como cal hidratada. El peor enemigo de la industria de la cal son muchos de los propios fabricantes, que con la pérdida gradual y constante del mercado ante el cemento y la competencia entre ellos, hacen un flaco favor a su producto al comercializarlo muy pobre en hidróxido de calcio, con la expectativa de ofrecer un producto denominado cal, que maximice sus utilidades en el marco de un mercado decadente y competido, sin que el desprestigio que ello acarrea amerite rectificar la calidad y pureza, de manera que mejore la percepción en los constructores de las cualidades que sí aporta el hidróxido de calcio a la industria de la construcción.

Tradicionalmente es bien conocido que la cal es un cementante débil comparado con el cemento, principalmente si ha sufrido una adulteración que demerita el contenido real de hidróxido de calcio. Consecuentemente, se debe emplear con cuidado en la construcción, ya que como conglomerante de escasa resistencia mecánica, un déficit de la misma tendría como consecuencia un importante debilitamiento y pérdida de durabilidad del mortero de cal. La pregunta que nos debemos formular es: ¿hasta qué punto es consciente el albañil o el operario que utiliza la cal de su pureza y la corrección que debe realizar? Ante esta incertidumbre, parece ra- 
zonable el establecimiento de una pureza mínima de cal para construcción como garantía tal y como hace la Norma Mexicana NMX-C-003-ONNCCE-1996, que debería cambiar su estatus de voluntaria a obligatoria.

Aún cuando se trata de una sustancia conocida por diversas culturas desde la antigüedad y que ha sido empleada por los romanos en construcciones ahora milenarias y por culturas mesoamericanas, como el principal aglomerante en construcciones o como aditivo fundamental en la cultura del maíz en la nixtamalización, la cal es un material con importantes aplicaciones en la industria moderna de la construcción, en la conservación y la restauración del patrimonio cultural, en la industria alimentaria y recientemente en nanotecnología, con materiales tales como nano-partículas de cal coloidal para consolidación de argamasa y mampostería (Ziegenbalg, 2008, Paiva et al., 2010). Por ello, un conocimiento más profundo de las transformaciones químicas e interacciones durante cada fase del ciclo de la cal es fundamental para renovar su vigencia y ampliar sus aplicaciones potenciales.

\section{Difracción de rayos $X(X R D)$}

En la figura 1a se muestra el patrón de difracción de rayos $X$ de la muestra $S A 1$, compuesta principalmente por calcita $\left(\mathrm{CaCO}_{3}\right)(\mathrm{PDF} 5-586)$, que presentan una estructura cristalina hexagonal. También se registraron señales correspondientes a la contribución de cristales de cuarzo $\left(\mathrm{SiO}_{2}\right)$ (PDF. 46-1045) al patrón de difracción, así como de cristales de muscovita $((\mathrm{K}, \mathrm{Na})$ $\left.(\mathrm{Al}, \mathrm{Mg}, \mathrm{Fe})_{2}\left(\mathrm{Si}_{3} \mathrm{AlO}_{9}\right) \mathrm{O}_{10}(\mathrm{OH})_{2}\right)$ (PDF 07-0042), de fluorita $\left(\mathrm{CaF}_{2}\right)(\mathrm{PDF} 35-0816)$ y de caolinita $\left(\mathrm{Al}_{2} \mathrm{Si}_{2} \mathrm{O}_{5}(\mathrm{OH})_{4}\right)$ (PDF 06-0221). La figura $1 \mathrm{~b}$ presenta el patrón de difracción de SA2. La muestra se obtuvo después del proceso de calcinación en horno vertical de combustóleo a temperaturas de entre 900 y $1200^{\circ}$ C. Durante este proceso la calcita sufre cambios: pierde el $44 \%$ de su peso, debido a su transformación química, en la cual se genera $\mathrm{CO}_{2}$ en fase gaseosa y se producen cristales de óxido de calcio $(\mathrm{CaO})$ con estructura cúbica. El óxido de calcio es muy hidrófilo, reacciona inmediatamente al entrar en contacto con la humedad del ambiente, produciendo pequeñas cantidades de hidróxido de calcio $\left(\mathrm{Ca}(\mathrm{OH})_{2}\right)$ (PDF 44-1481) de fase hexagonal, cuyos cristales incrementan su tamaño paulatinamente con el paso del tiempo, lo que conlleva a un decaimiento en la reactividad del óxido de calcio. Por esta razón, están presentes cristales de portlandita $\left(\mathrm{Ca}(\mathrm{OH})_{2}\right)$ de fase hexagonal en esta muestra. El patrón de difracción también presenta señales correspondientes a trazas de calcita, la cual principalmente es un remanente del proceso de calcina- ción. El centro de las rocas más grandes no se calienta lo suficiente para poder reaccionar durante la calcinación y por ello, se conserva una cantidad de calcita. También, la calcita podría ser el producto de la reacción de carbonatación del $\mathrm{Ca}(\mathrm{OH})_{2}$ que se ha formado, pero esta reacción requiere un tiempo mayor, por lo que serían cantidades muy pequeñas de calcita que se formarían por esta vía. Además, en el patrón se identificaron cristales de cuarzo $\left(\mathrm{SiO}_{2}\right)$ con estructura cristalina hexagonal, así como cristales de larnita $\left(\mathrm{Ca}_{2} \mathrm{SiO}_{4}\right)$ de fase monoclínica. Esta última fase cristalina es un componente poco abundante en el material proveniente de la cantera, de la cual se extraen las rocas con mineral de carbonato de calcio, su distribución es muy heterogénea dentro de la cantera y eso hace que su aparición varíe de lote a lote de rocas, y es por ello que su presencia es variable, no se registró su presencia en SA1 y SA3, pero si en la muestra SA2.

La presencia de azufre, un elemento que no es común en la composición de la materia prima, como muestra en el patrón de difracción de la muestra SA1, se considera un contaminante, mismo que proviene del combustible utilizado durante el proceso de calcinación.

Finalmente, la figura 1c muestra el patrón de difracción de SA3, que corresponde a hidróxido de calcio. Nuevamente, este patrón permite observar los cambios químicos y cristalográficos en la evolución del ciclo de la cal: luego de la hidratación del óxido de calcio, la cual se produce por medio de una violenta reacción química exotérmica, se generan los cristales de portlandita

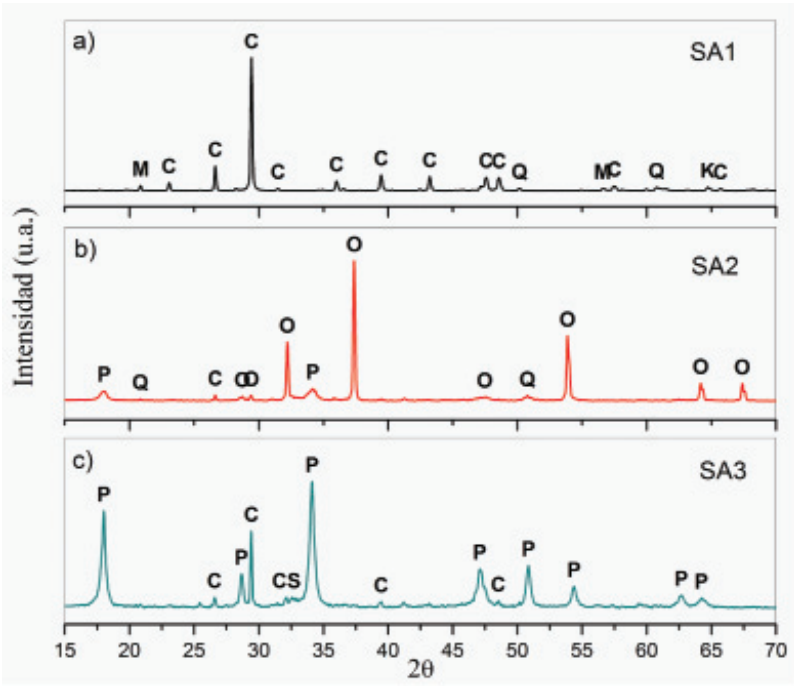

Figura 1. a) patrón de difracción de rayos $X$ de $S A 1$, b) patrón de SA2, c) patrón de SA3. Nomenclatura: C-calcita, Q-cuarzo, K-caolinita, M-muscovita, O-óxido de calcio, P-portlandita 
$\left(\mathrm{Ca}(\mathrm{OH})_{2}\right)$ con estructura hexagonal. En el patrón, también se registraron señales correspondientes a cristales de calcita, los cuales son el remanente de la materia prima empleada, cristales de cuarzo $\left(\mathrm{SiO}_{2}\right)$ en baja cantidad, según la intensidad de sus señales y cristales de silicato de cuarzo $\left(\mathrm{Ca}_{2}\left(\mathrm{SiO}_{4}\right)\right)$ (PDF 86-0398) y anhidrita $\left(\mathrm{Ca}\left(\mathrm{SO}_{4}\right)\right)$ (PDF 37-1496); esta última posiblemente originada por el azufre proveniente del combustible, el cual al entrar en contacto con el agua de la hidratación reacciona para formar óxido de azufre $\left(\mathrm{SO}_{2}\right)$ y éstos posteriormente reaccionan con el carbonato de calcio para formar la anhidrita. De acuerdo con estos resultados, las fases minerales pueden variar de muestra a muestra desde su origen en la cantera; por esta razón, una semicuantificación de estas fases tendría poco sentido.

\section{Microscopía electrónica de barrido (SEM)}

La superficie de cada muestra fue cuidadosamente observada de manera general antes de que las fotografías fueran obtenidas, de tal forma que las imágenes presentadas fueran representativas de cada muestra, mostrando la morfología de las fases minerales presentes y las características topológicas de la misma. Las figuras 2a, 2 b y 2 c son fotografías de las muestras SA1, SA2 y SA3, respectivamente. La muestra SA1 está constituida por grandes cristales, principalmente de calcita con superficies lisas. Se observa una morfología definida de los cristales con bordes y aristas bien delimitados. En la figura $2 \mathrm{a}$, se pueden observar algunos estratos en los cristales. En la superficie de esta muestra no se encontraron poros y las grietas visibles son el producto de la manipulación desde el proceso de muestreo hasta la preparación de la muestra. En general, se observa un

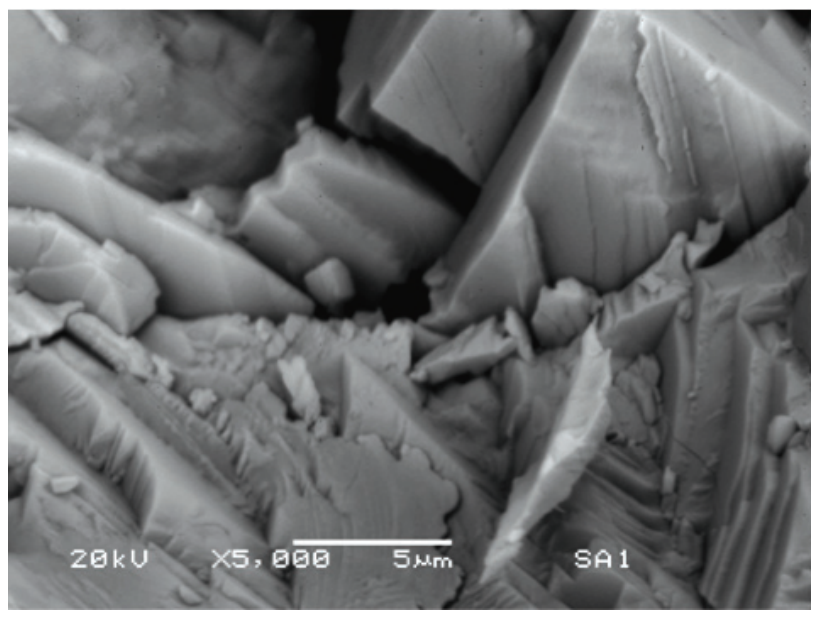

Figura 2a. Muestra SA1, carbonato de calcio

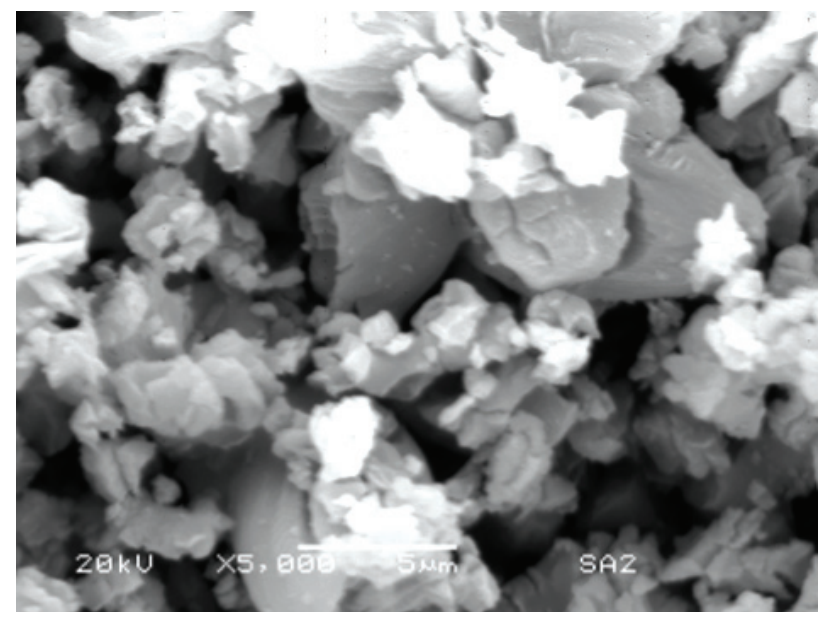

Figura 2b. Muestra SA2, óxido de calcio

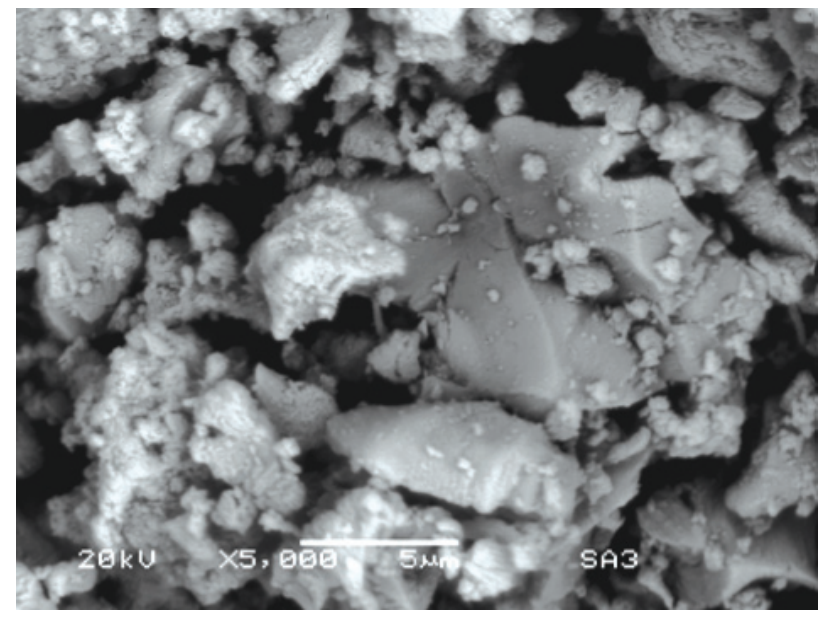

Figura 2c. Muestra SA3, hidróxido de calcio

buen crecimiento cristalino, el cual se llevó a cabo de manera natural durante muchos años.

La muestra SA2 presenta partículas pequeñas con una distribución amplia de tamaños de partículas, la cual va desde la escala nanométrica $(800 \mathrm{~nm})$ hasta el orden de decenas de micrómetros $(20 \mu \mathrm{m})$. En este material se llevó a cabo un proceso de molienda sin efectuar un filtrado por tamiz y la calcinación para transformar la calcita a óxido de calcio. Los bordes de las partículas están bien definidos, pero no tienen una forma en particular; su morfología es básicamente un remanente de aquella que presentaba la calcita antes de la calcinación. En la muestra SA3 se encontraron partículas más pequeñas. La distribución de tamaños para esta muestra también es muy amplia, pero predominan los tamaños pequeños en el orden de los 300 a los $60 \mathrm{~nm}$, aunque fue posible observar partículas de hasta $15 \mu \mathrm{m}$. 
La reducción del tamaño promedio de partículas se debió a que la reacción de hidratación del óxido de calcio es muy violenta, liberando una gran cantidad de energía en breve tiempo. Muchas de las partículas de óxido de calcio se fragmentan, debido a esta reacción, produciendo partículas de hidróxido de calcio de menor tamaño. En la figura 2c se puede observar una partícula grande con grietas en su superficie, las cuales se formaron por el efecto de la reacción de hidratación. Las partículas pequeñas aparentan tener una superficie rugosa y porosa, la cual también puede ser el producto de la reacción exotérmica.

\section{Espectroscopía infrarroja por transformada de Fourier (FTIR)}

FTIR es una técnica analítica útil que permite identificar y cuantificar componentes de una mezcla y, a diferencia de la difracción de rayos $X$, permite el análisis de compuestos orgánicos o inorgánicos no cristalinos. El análisis cualitativo de las frecuencias características proporciona información para identificar los componentes químicos en una mezcla. El análisis por FTIR resulta complementario al análisis hecho por difracción de rayos X. La figura 3a muestra el espectro de FTIR para la muestra SA1. La banda a $3630 \mathrm{~cm}^{-1}$ corresponde a la vibración de los grupos $\mathrm{O}-\mathrm{H}$, los cuales se relacionan con la presencia de una baja concentración de $\mathrm{Ca}(\mathrm{OH})_{2}$ en la muestra; es una señal intensa, como es habitual para este tipo de enlaces y debido a su forma estrecha, por lo que podemos afirmar que la muestra contiene muy poca humedad absorbida. El hidróxido es un compuesto remanente del proceso de carbonatación. Las bandas a 1440, 875 y $713 \mathrm{~cm}^{-1}$ corresponden a los tres modos diferentes de vibración del enlace $\mathrm{C}=\mathrm{O}$ contenido en el ión carbonato mientras las bandas a 2980, 2875, 2513 y $794 \mathrm{~cm}^{-1}$ corresponden a vibraciones armónicas de estos modos de elongación (Farcas, 2001). $\mathrm{El}$ ancho de estas señales es evidencia de que los grupos $\mathrm{C}=\mathrm{O}$ deben estar formando puentes de hidrógeno. Las bandas a $2875 \mathrm{~cm}^{-1}$ y el primer sobretono a $2980 \mathrm{~cm}^{-1}$, aparecen a una intensidad mayor a la reportada por Nyquist et al. (1996). La banda intensa en $1795 \mathrm{~cm}^{-1}$ está asociada con el enlace $\mathrm{C}-\mathrm{O}$ del ión carbonato. La banda más intensa y ancha del espectro en $1444 \mathrm{~cm}^{-1}$ brinda evidencia de los iones carbonato. Las bandas a 1105, 1018 y $790 \mathrm{~cm}^{-1}$ están relacionadas con $\mathrm{SiO}_{2}$ (cuarzo), confirmado por los análisis XRD ya discutidos. La banda a $713 \mathrm{~cm}^{-1}$ está relacionada con el enlace $\mathrm{Ca}-\mathrm{O}$. $\mathrm{Al}$ comparar los espectros de SA1 con los de un estándar de $\mathrm{CaCO}_{3}$ (Baker analized 99.9\%), la señal intensa a $875 \mathrm{~cm}^{-1}$ está relacionada al ión $\mathrm{CO}_{3}(\mathrm{Keum}, 2003)$ y es más intensa para el estándar que para SA1, pero al comparar el resto de los espectros entre la muestra y el estándar, se observa una gran similitud. En la figura $3 \mathrm{~b}$ se muestran los espectros de SA2 y el estándar de espectros de $\mathrm{CaO}$ (Fermont - CAS 1305-78-8). La banda intensa a $3643 \mathrm{~cm}^{-1}$ se corresponde con el enlace $\mathrm{O}-\mathrm{H}$ del hidróxido de calcio (Park et al., 2002). El pico en la banda $1417 \mathrm{~cm}^{-1}$ corresponde a enlaces $\mathrm{C}-\mathrm{O}$. La banda a $866 \mathrm{~cm}^{-1}$ también corresponde a los grupos C-O. La señal intensa y ancha al alrededor de $500 \mathrm{~cm}^{-1}$ pertenece al enlace de $\mathrm{Ca}-\mathrm{O}$, que es la principal señal para la identificación del óxido de calcio $\mathrm{CaO}$. En este espectro también se observan las señales a $1440 \mathrm{~cm}^{-1}$ correspondientes a la calcita y señales a 1105, 1018 y $790 \mathrm{~cm}^{-1}$ que identifican al cuarzo. La calcita es un remanente del proceso de calcinación, mientras que el cuarzo permanece inerte durante esta reacción química. La figura $3 c$ muestra los espectros de SA3 y del estándar de $\mathrm{Ca}(\mathrm{OH})_{2}$. La banda fuerte a $3643 \mathrm{~cm}^{-1}$ está relacionada con el enlace O-H de hidróxidos. La banda a $1795 \mathrm{~cm}^{-1}$ corresponde al enlace de carbonato $\mathrm{C}-\mathrm{O}$. La banda intensa y ancha en $1440 \mathrm{~cm}^{-1}$ corresponde a los grupos $\mathrm{C}=\mathrm{O}$ del carbonato, su intensidad es producto de la abundancia de estos grupos. Lo anterior es evidencia de la transformación del hidróxido de calcio, el cual reacciona al entrar en contacto con el dióxido de carbono del ambiente para producir carbonato de calcio. La banda a $875 \mathrm{~cm}^{-1}$ también corresponde a los grupos carbonato. La banda débil a $1126 \mathrm{~cm}^{-1}$ correspondiente a los enlaces S-O y revela la presencia de óxido de azufre, contaminante

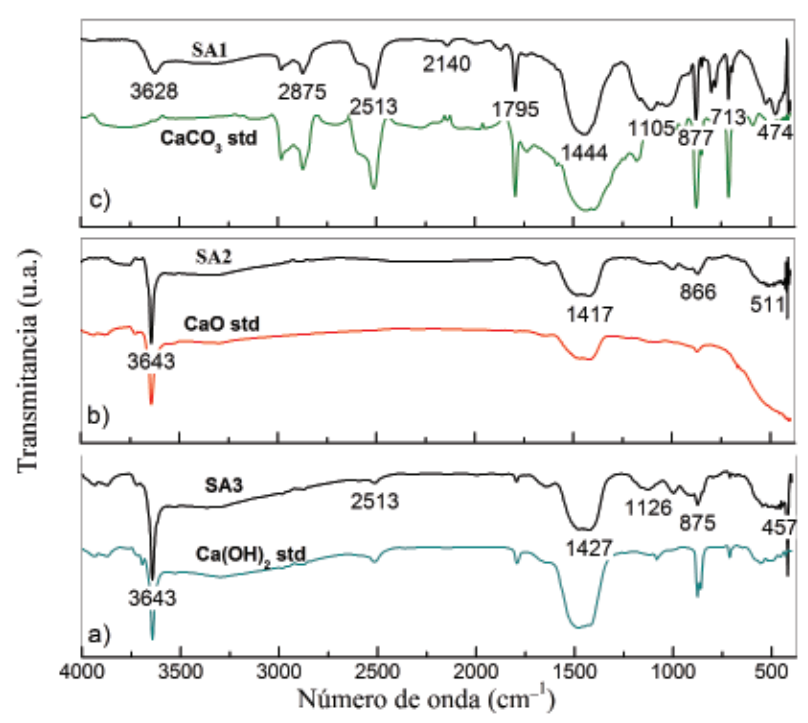

Figura 3. a) FT-IR de SA1 (arriba), estándar de $\mathrm{CaCO}_{3}$ (abajo). b) FT-IR de SA2 (arriba), estándar de $\mathrm{CaO}$ (abajo). c) FT-IR de SA3 (arriba), estándar de $\mathrm{Ca}(\mathrm{OH})_{2}$ (abajo) 
posiblemente añadido durante el proceso de calcinación por la ignición de combustibles fósiles pesados. La banda en la región de $500 \mathrm{~cm}^{-1}$ corresponde a los enlaces $\mathrm{Ca}-\mathrm{O}$. Los espectros del $\mathrm{Ca}(\mathrm{OH})_{2}$ estándar (Fermont $98,6 \%$ ), se asocian estrechamente con el espectro de SA3. En resumen, es razonable afirmar que el $\mathrm{CaCO}_{3}$ fue obtenido de una cantera mexicana y se transformó en $\mathrm{CaO}$, a través de un proceso de calcinación. Esta reacción tiene una baja eficiencia y, en consecuencia, el $\mathrm{CaO}$ obtenido estaba acompañado de cantidad considerable de $\mathrm{CaCO}_{3}$ además de cantidades pequeñas de otros minerales como el cuarzo. Estos minerales secundarios no sufrieron ninguna transformación química durante la reacción de calcinación ni en las reacciones posteriores. La ignición de combustible derivado del petróleo produjo gases de óxido de azufre durante la reacción de calcinación, y estos compuestos pueden contaminar el producto con compuestos de azufre; la presencia de estos contaminantes está relacionada con la calidad de los combustibles utilizados. Si se utiliza un combustible rico en compuestos orgánicos sulfurados, el $\mathrm{CaO}$ producido tendrá mayor contenido de compuestos de azufre.

\section{Espectrometría de masas por inducción de plas- ma acoplado (ICP-MS)}

En las muestras se analizaron concentraciones de varios elementos potencialmente peligrosos para la salud y algunos isótopos. El equipo permite detectar concentraciones de hasta partes por trillón. Luego del análisis por espectrometría de masas para evaluar metales pesados, se encontró que las tres muestras analizadas en este estudio contienen elementos químicos peligrosos que no significan riesgos de acuerdo con las normas, tanto nacionales como internacionales, cuya consideración aporta una mejor visión del horizonte de análisis; los resultados se muestran en la tabla 2. La presencia de los elementos detectados mediante ICP-MS en las muestras, no tiene mayor efecto para el uso de la cal en la construcción porque su concentración es prácticamente imperceptible. No obstante, es interesante mati- zar el contenido de metales pesados y otras substancias peligrosas porque la cal hidratada, aunque fabricada primordialmente para su uso en la construcción, se utiliza también ampliamente en el proceso de nixtamalización del maíz; de acuerdo con datos de la ANFACAL, se usan en el orden de 100,000 toneladas anualmente para nixtamalización; luego de verificar la Norma Oficial Mexicana NOM 187-SSA1/SCSI-2002 relativa a masa, tortillas, tostadas y harinas preparadas para su elaboración, las muestras analizadas se encuentran dentro de los límites en contenido de metales pesados y otros elementos peligrosos, aceptables para consumo humano, ello a pesar del uso de combustóleo para la calcinación y de agua sin control sanitario para la hidratación.

\section{Dispersión dinámica de luz}

La distribución del tamaño de partículas se muestra en la figura 4. Es interesante destacar que la totalidad de partículas tienen un tamaño inferior a 1 micra con: $931.49 \mathrm{~nm}$ (41 partículas), 853.10 (46), 781.31 (44), 327.48 (9), 323.83 (47), 320.22 (86), 316.65 (100), 313.12 (61), 309.63 (23), 133.35 (48), 100 (81), 74.99 (100), 56.23 (57), y 42.17 (24 partículas). Aquí es posible establecer que cuando se realizó la segunda medición, las partículas más pesadas se precipitaron a la parte inferior del vial y más partículas ligeras contribuyeron con mayor intensidad en la distribución de los tamaños. Finalmente en la tercera medición, partículas aún más ligeras que las observadas en la medición anterior, contribuyeron a una muy precisa determinación de la distribución de los tamaños de partículas. La distribución de tamaño de partícula es pertinente porque demuestra que el hidróxido de calcio contiene una significativa fracción de nanopartículas. Esto es relevante porque las partículas se originan naturalmente luego de una violenta reacción exotérmica durante la hidratación del óxido de calcio, a diferencia de las partículas de cemento Pórtland, que se originan luego de la molienda del clinker y el tamaño de sus partículas se encuentra en un rango entre 5 y $100 \mu \mathrm{m}$, mucho mayor que el de la cal. Esto explica por qué la cal tiene propiedades superiores al

Tabla 2. Determinación del contenido de metales pesados por espectrometría de masas

\begin{tabular}{|c|c|c|c|c|c|c|c|c|c|c|c|c|}
\hline \multirow{2}{*}{ Muestra } & \multicolumn{12}{|c|}{ Concentración en mg/kg } \\
\hline & $\mathrm{Li}$ & Be & $\mathrm{V}$ & $\mathrm{Cr}$ & Co & $\mathrm{Ni}$ & $\mathrm{Cu}$ & As & Se & $\mathrm{Cd}$ & $\mathrm{Tl}$ & $\mathrm{Pb}$ \\
\hline SA1 & 1.3669 & 0.0 & 1.0211 & 0.0481 & 1.2674 & 2.3924 & 0.6621 & 0.4008 & 0.0 & 0.0 & 0.0536 & 0.8076 \\
\hline SA2 & 0.0087 & 0.0 & 1.9068 & 0.1066 & 0.0686 & 1.4785 & 0.2742 & 0.0290 & 0.0 & 0.0 & 0.0 & 0.0012 \\
\hline SA3 & 0.3401 & 0.0 & 4.3403 & 0.2150 & 0.0884 & 1.1589 & 0.1932 & 0.0849 & 0.0 & 0.0 & 0.0 & 0.0527 \\
\hline
\end{tabular}




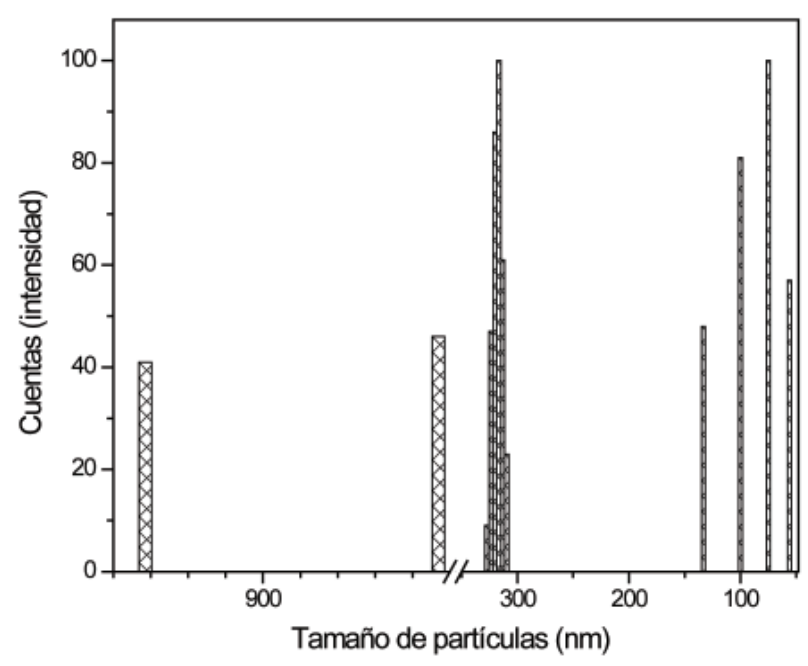

Figura 4. Distribución de los tamaños de partículas del hidróxido de calcio

cemento en cuanto a impermeabilidad, capilaridad, porosidad, difusión térmica y acústica, contracción química y autógena, y evolución de humedad interna, además de mejor adherencia. Aunque el hidróxido de calcio es uno de los materiales de arte y construcción de mayor antigüedad que ha utilizado el hombre, poco se ha estudiado acerca de su nano-estructura y características coloidales, que desempeñan un papel crucial en su máximo rendimiento como componente en morteros de cal y yeso. Particularmente, se desconoce por qué la argamasa de cal hidratada se comporta como un coloide irreversible una vez que pierde la humedad. Este efecto afecta considerablemente la reactividad y la reología de dispersión de la cal hidratada (Rodriguez et al., 2005).

\section{Conclusiones}

Los resultados de los análisis llevados a cabo por medio de las diferentes técnicas de caracterización utilizadas en este trabajo de investigación han permitido comprobar, por una parte, las diferentes etapas de transformación que experimentan los materiales dentro del ciclo de la cal, y la reactividad de los componentes químicos que se forman en cada etapa. Se comprobó la naturaleza heterogénea en composición química que presenta una mina de piedra caliza y la relativa abundancia de elementos pesados dentro de una producción típica de hidróxido de calcio. Los resultados de esta investigación permiten sugerir que la Norma Mexicana NMXC-003-ONNCCE-1996 deje de ser voluntaria y en su lugar se establezca una norma que regule la calidad de la producción de hidróxido de calcio.
El estudio de la distribución de los tamaños de partículas del hidróxido de calcio demuestra que la cal hidratada podría ser uno de los primeros nano-materiales desarrollados y utilizados por el hombre.

\section{Agradecimientos}

Agradecemos sinceramente el apoyo técnico de Leticia Baños, Alicia del Real, Ofelia Pérez y Carolina Muñoz, así como al Centro de Física Aplicada y Tecnología Avanzada (CFATA) de la Universidad Nacional Autónoma de México y a la Asociación Nacional de Fabricantes de Cal (ANFACAL), a quienes expresamos nuestro reconocimiento.

\section{Referencias}

Arandigoyen M. y Álvarez J. Proceso de carbonatación en pastas de cal con distinta relación agua/conglomerante. Materiales de Construcción, 56(281):5-18. 2006.

Cazalla O., Rodríguez-Navarro C., Sebastián E., Cultrone G., Torre M. Aging of Lime Putty: Effects on Traditional Lime Mortar Carbonation. Journal of the American Ceramic Society, 83(5):1070-1076. 2000

Cowper A. Lime and Lime Mortars. UK: Donhead Publishing LTD. 1927. Reprinted 2000.

Delatte N. Lessons from Roman Cement and Concrete. J. Profl. Issues in Engrg. Educ. and Pract.,: 127, 3:109-115. 2001.

Farcas F. La Spectrométrie Infrarouge à Transformée de Fourier (IRTF). Une Méthode Intéressante pour la Caractérisation des Ciments. Bulletin des Laboratoires des Ponts et Chaussées, 230:7778. 2001.

Hill N. Holmes S., Mather D. Lime and Other Alternative Cements. UK: Intermediate Technology Publications. 1992.

Holmes S., Wingate M. Building with Lime. UK: ITDG Publishing. 2003.

Keum D., Naka K., Chujo Y. Unique Crystal Morphology of Hydrophobic Composite by Sodium Trisilanolate in a Mixture of a Water-Miscible Organic Solvent and Water. Journal of Crystal Growth, 259:411-418. 2003.

Moropoulou A., Bakolas A., Aggelakopoulou E. The Effects of Limestone Characteristics and Calcination Temperature to the Reactivity of the Quicklime. Cement and Concrete Research, 31:633-639. 2001.

Moropoulou A., Cakmakb A., Labropoulosa K., Van Griekenc R., Torfsc K. Accelerated Microstructural Evolution of a CalciumSilicate-Hydrate (C-S-H) Phase in Pozzolanic Pastes Using Fine Siliceous Sources: Comparison with Historic Pozzolanic Mortars. Cement and Concrete Research, 34:1-6. 2004.

Moropoulou A., Polikreti K., Bakolas A., Michailidis P. Correlation of Physicochemical and Mechanical Properties of Historical Mortars and Classification by Multivariate Statistics. Cement and Concrete Research, 33:891-898. 2003. 
Nyquist R, Putzig C., Leugers M. Infrared and Raman Spectral Atlas of Inorganic Compounds and Organic Salts. USA, Boston Academic. 1996.

Paiva H., Velosa A., Veiga R., Ferreira V. Effect of Maturation Time on the Fresh and Hardened Properties of an Air Lime Mortar. Cement and Concrete Research, 40(3):447-451. 2010.

Park J., Min D., Song H. Structural Investigation of $\mathrm{CaO}-\mathrm{Al}_{2} \mathrm{O}_{3}$ and $\mathrm{CaO}-\mathrm{Al}_{2} \mathrm{O}_{3}-\mathrm{CaF}_{2}$ Slags Via Fourier Transform Infrared Spectra. ISIJ International, 42(1):441-445. 2002.

Rodriguez-Navarro C., Ruiz-Agudo E., Ortega-Huertas M., Hansen E. Nanostructure and Irreversible Colloidal Behavior of
$\mathrm{Ca}(\mathrm{OH})_{2}$ : Implications in Cultural Heritage Conservation. Langmuir, 10948-10957. 2005.

Shi C. Studies on Several Factors Affecting Hydration and Properties of Lime-Pozzolan Cements. J. Mat. in Civ. Engrg., 13(6):441445. 2001.

Ziegenbalg G. Directed and Controlled Crystallisation of Slightly Soluble Minerals - A New Technology to Seal Water Inflows and to Immobilise Contaminants. Proceedings of the 10th IMWA Congress. Editors: Rapantova, N., Hrkal, Z, pp. 55-60, 2008.

\section{Semblanza de los autores}

Miguel Galván-Ruiz. Doctor en ingeniería. Trayectoria docente de más de tres décadas en los niveles de bachillerato, licenciatura y maestría, con adscripción actual en la Facultad de Ingeniería de la Universidad Autónoma de Querétaro. Encabeza un grupo de investigación y es responsable de dos proyectos financiados. Ha sido director de 6 tesis de maestría, 3 de licenciatura y consultoría científica relacionada con la industria de la cal y con materiales para restauración en sitios declarados patrimonio histórico supervisados por el INAH. Revisor de proyectos, autor de 6 artículos en revistas internacionales arbitradas. Miembro del Sistema Nacional de Investigadores (SNI) 2010-2012, nivel C.

Rodrigo Rafael Velázquez-Castillo. Nacido en la ciudad de México, se graduó en química por la Facultad de Química en 1990. Posteriormente estudió la maestría en ciencia de materiales en la Facultad de Ciencias y obtuvo el doctorado en ciencias químicas en la Facultad de Química en 2001, todo en la Universidad Nacional Autónoma de México. Llevó a cabo una estancia posdoctoral en el Texas Materials Institute de la Universidad de Texas en Austin en 2005. Ha colaborado en instituciones como la Universidad de Guadalajara, la UNAM, la Universidad Autónoma de Querétaro, la Universidad del Valle de Atemajac y el ITESM. Pertenece al SNI nivel I. 